\title{
INSPECTION PLATFORM FOR APPLICATIONS IN HUMANITARIAN DEMINING
}

\author{
H. MONTES ${ }^{1,2}$, L. MENA ${ }^{1}$, R. FERNANDEZ ${ }^{1}$, J. SARRIA ${ }^{1}$ and M. ARMADA ${ }^{1}$ \\ ${ }^{I}$ Field and Service Robotics Group, Centre for Automation and Robotics-CAR (CSIC- \\ UPM), Spain; ${ }^{2}$ Faculty of Electrical Engineering, Technological University of Panama, \\ Panama, hector.montes@,csic.es
}

\begin{abstract}
The inspection platform that is described in this manuscript consists of a hexapod walking robot designed by the Centre for Automation and Robotics CSIC-UPM, Spain. This inspection platform will load a scanning manipulator arm which, in turn, carries a metal detector on its tool centre point. With the integration of both, hexapod robot and scanning manipulator, several test tasks about the search and localisation of antipersonnel mines will be carried out, within a controlled environment. The SCARA configuration of the hexapod robot legs will allow low energy consumption when the robot executes gaits on flat terrain or with reduced slope, due the decoupling of gravitational effects. This legged robot has a mass about $250 \mathrm{~kg}$, and it can bear a high payload up to about $300 \mathrm{~kg}$. Considering this load characteristic then the vibrational effects on the scanning manipulator will be reduced, when this carry out scanning tasks over the terrain.
\end{abstract}

\section{Introduction}

Detection and removal of landmines fields is a severe problem with amazing political, social and economic dimensions, which have been present during the last decades [1]. In this context, the international scientific community has shown a broad interest to solve this problem, which is addressed from different points of view and with different procedures. The anti-personnel mines, submunitions and unexploded ordnance (UXO) can remain active for several years (in some cases up to 50), they do not can discriminate between military and civilian people, killing or injuring indiscriminately to them, including children and humanitarian collaborators [2].

Currently, it is estimated that there are 110 million landmines buried in the ground, and more than 160 million are in stockpiles waiting to be planted or destroyed [3]. One solution to this problem could be the application of fully automatic systems or tele-operated systems to detect and remove the landmines. However, regardless of the latest advances in this field, this solution still seems far from possible. 
In order to solve this problem, using the above mentioned systems, should be considered efficient sensors, detectors and positioning systems for detecting, locating and to identify the landmines. Besides, appropriate mobile vehicles must be developed in order to have on board the sensors for move them on infested fields of mines and, in this way, put off to human operator of a direct risk. Additionally, should be interesting to have a human-robot collaboration to carry out the humanitarian demining tasks, since this work is very complex and difficult to realize.

Many types of mobile vehicles that can take on board sensors to move them to a mine-infested area; e.g., wheeled vehicles, tracked vehicles and even legged robots; can carry out demining tasks efficiently and safely, relatively. Wheeled robots are the simplest and least costly [4-7]; tracked robots have excellent ability to travel on almost any terrain $[1,8,9]$; however, legged robots have a very interesting potential for this activity [10-15].

Legged robots for carry out humanitarian demining tasks have been developed, at least, in the last 15 years, and several prototypes of these robots have been tested experimentally. Some examples of these robotics platforms are TITAN VIII [12], AMRU-2 [11], RIMHO2 [10], COMET series [14], SILO6 $[13,15]$. These walking robots are based on configurations of legs insect type. But have been developed other configurations of walking robots for demining, such as sliding frame systems $[11,16]$. The need to develop legged robots to operate in certain situations where other humanitarian demining systems using wheels or tracks cannot operate properly or have low yield, has been investigated by several research centres (Roya Military Academy (RMA), Free University of Brussels, Chiba University, Tokyo Institute of Technology, The Spanish National Research Council (CSIC), other).

In this work the main characteristics of a hexapod robot designed and manufactured by the Centre for Automation and Robotics (CAR) CSIC-UPM are presented. The main objective of this hexapod walking robot is carry out tasks for localisation of antipersonnel mines, taking on board a scanning manipulator arm with a metal detector installed on its tool centre point. Primarily, Section 2 describes the mechanical design and system architecture of the hexapod robot. Afterwards, Section 3 presents some experimental results carried out on flat surface within a controlled environment. Finally, conclusions are given in Section 4. 


\section{General configuration of the hexapod robot}

The hexapod walking robot has been configured to carrying out tasks of humanitarian demining according to the requirements established in TIRAMISU Project ${ }^{*}$. This legged robot will carry on-board a scanning manipulator of $5 \mathrm{DoF}$, several sensors to help the scanning tasks, the industrial computer, electronics, control/power cards, Wi-Fi wireless communication system, DGPS, batteries, and other devices and accessories.

\subsection{Description of the mechanical system}

The hexapod robot legs have a SCARA configuration (RRP). This configuration decouples gravitationally the movement of the robot body (obviously, when it is walking on surfaces without slope). For this reason, this hexapod robot has an energetic autonomy relatively high, considering its mass $(\sim 250 \mathrm{~kg})$ and the payload that it can to load $(<300 \mathrm{~kg})$. Therefore, the scanning manipulator arm with metal detector and other sensors and devices installed on-board of this hexapod robot shall not comprise a significant load to itself. Figure 1 shows the mechanical configuration of the hexapod robot. Table 1 shows the main characteristics of this walking inspection platform.

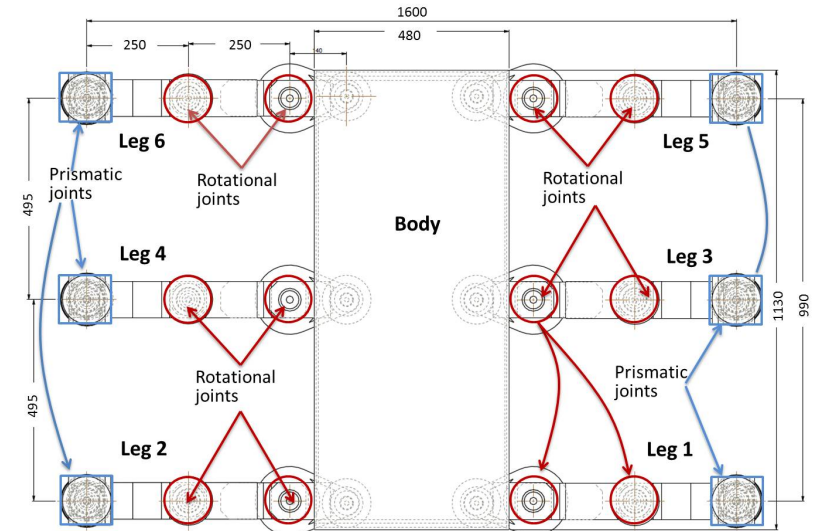

(a)

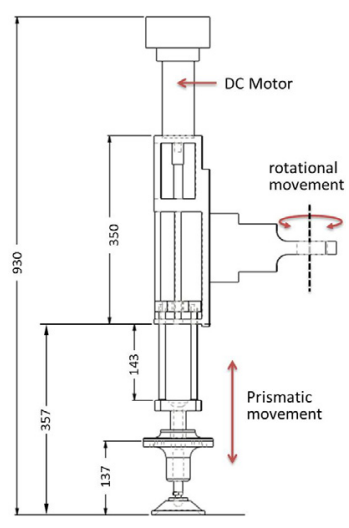

(b)

Figure 1. Mechanical configuration of the hexapod robot (units in $\mathrm{mm}$ ). (a) Top view of the robot; (b) Frontal view of the leg.

\footnotetext{
${ }^{*}$ http://www.fp7-tiramisu.eu/
} 
Table 1. Main mechanical characteristics of the hexapod walking robot.

\begin{tabular}{l|l|l}
\hline Degrees of freedom & 18 \\
\hline Stability & High \\
\hline Robot mass & $250 \mathrm{~kg}$ \\
\hline Payload capacity & Up to $300 \mathrm{~kg}$ \\
\hline \multicolumn{2}{l|}{ Obstacle height to surpass } & Up to $200 \mathrm{~mm}$ \\
\hline Power supply & $16-30 \mathrm{VDC}$, Typ. 24 VDC \\
\hline \multirow{2}{*}{ Body size } & Length & $1130 \mathrm{~mm}$ \\
\cline { 2 - 3 } & Width & $480 \mathrm{~mm}$ \\
\hline \multirow{2}{*}{ Robot size } & Max length & $1130 \mathrm{~mm}$ \\
\cline { 2 - 3 } & Max width & $1700 \mathrm{~mm}$ \\
\hline
\end{tabular}

The main robot gait is performed by means of an alternating tripod gait. However, other gait alternatives are being developed at the time of writing of this manuscript. The hexapod has three feet in contact with the ground all times, while the other legs are in transfer phase. In this gait two non-adjacent legs of one side and the central leg of the opposite side of the robot support the robotic platform, with high stability, while the other tripod is in the transfer phase [17].

\subsection{System architecture description}

The system architecture of the hexapod robot consist of an on-board computer, control cards, data acquisition boards, power cards, signals conditioner cards, positioning sensors, DC motors, Wi-Fi communication system, DGPS, batteries, and other devices and accessories. This control architecture provides a reliable starting point for developing several control strategies in order to carry out humanitarian demining tasks. Figure 2 shows the most important subsystems that comprise the control architecture assembled in the hexapod robot, and a perspective view of the robot [17].

The central idea is carry out stable gaits in order that scanning manipulator arm can perform suitable movements of its end effector, where is installed the metal detector head. In this regard, the inspection platform shall move to a fix position in order that the manipulator arm carries out the ground exploration. Standard strategies for the sweeping techniques will be considered. Some explanations related with these standards can be seen in [18]. According to the strategies of demining tasks (commanded by the operator), the displacement of the robot is proposed. These strategies are sent to the trajectory generator stage by mean of Wi-Fi communication, which uses the robot kinematics and the PID 
control to perform the controlled movement of the hexapod robot. Figure 3 shows the general control architecture of the hexapod robot.
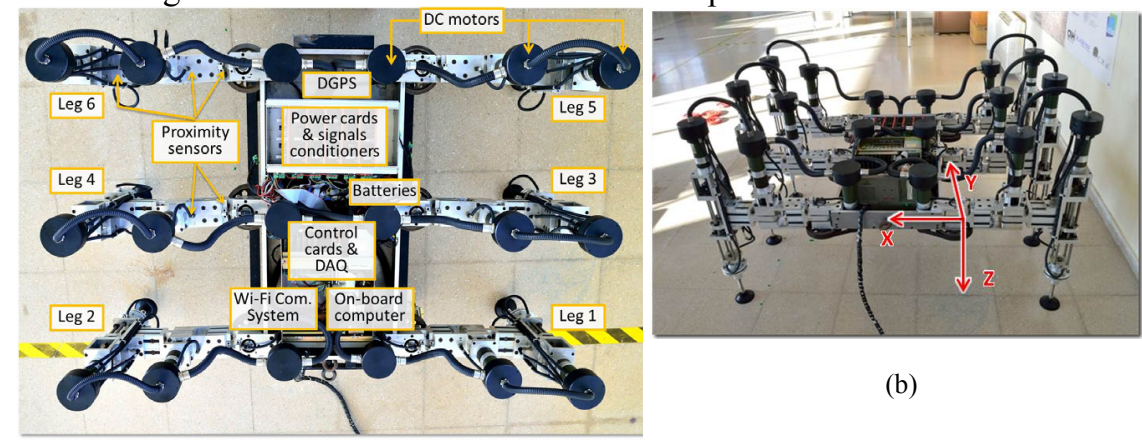

(b)

Figure 2. (a) Subsystems of the control architecture of the hexapod robot; (b) Hexapod walking robot in CAR (CSIC-UPM) lab.

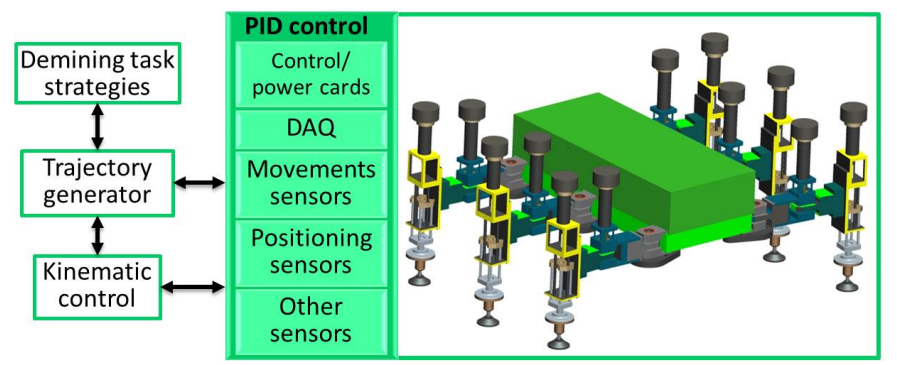

Figure 3. General control architecture of the hexapod robot.

\section{Experimental results}

Several experimental tests with different gait patterns have been carried out in the CSIC lab. The hexapod robot has demonstrated high stability during the following of several trajectories. This robot has moved loads of more than $50 \mathrm{~kg}$ during some tests, and the additional power consumption has been insignificant with respect to the execution of gait patterns without extra payload. Therefore, will be feasible to carry on-board the scanning manipulator and other additional sensors, and this do will not contribute to a significant energy consumption.

Figure 4 shows some experimental results during the execution of a continuous gait using the alternating tripod mode. In order to see some details of the experimental results only two steps are presented in Figure 4. Figures 4(a) and 4(b) show the position of the shoulder and elbow joints of the leg 1 and leg 2 , respectively. Each of these legs moves with different tripods. For this reason, these graphics represent the motion of each tripod, assuming that the 
corresponding legs of each tripod have similar motions. In Figure 4(b), backlash in elbow joint can be appreciated. Figure 3(c) shows the position of both legs along the $Z$ axis, the alternating gait is perceived, besides a small unevenness on the soil is detected. Figure 4(d) show the lateral displacement of the extreme leg 1 , the commanded straight line has an error about $\pm 0.51 \mathrm{~mm}$, wherewith is considered reasonably right.
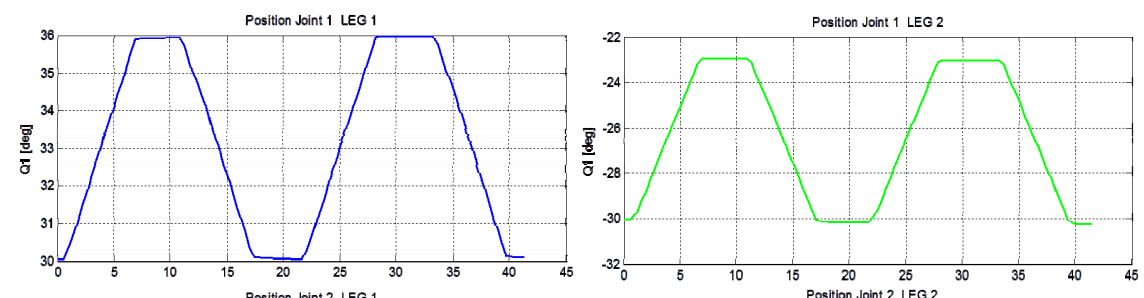

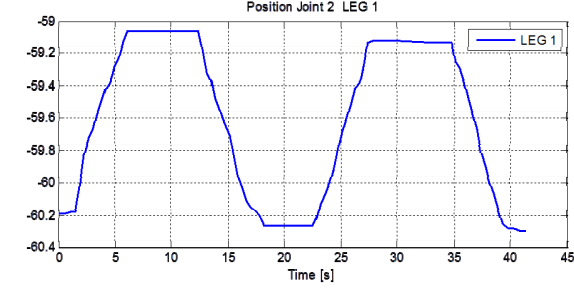

(a)

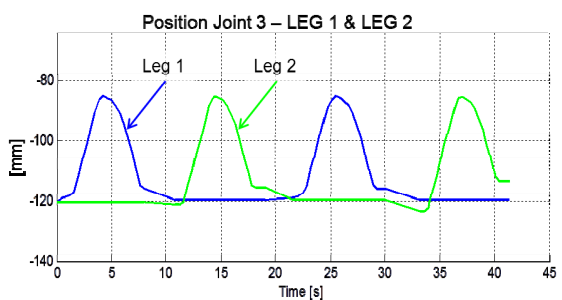

(c)

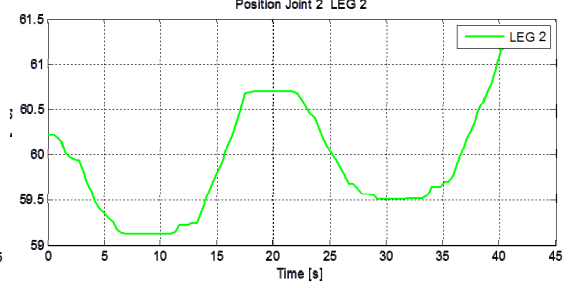

(b)

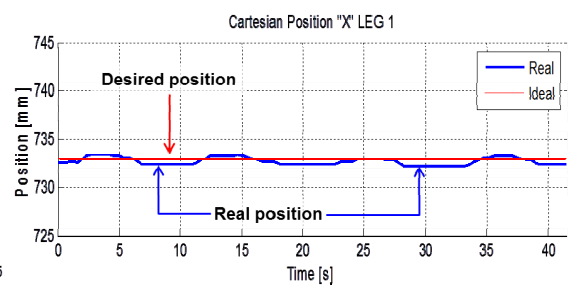

(d)

Figure 4. Some experimental results during the execution of a continuous gait using the alternating tripod mode.

The transfer and stance phases of the leg 1during two steps are shown in Figure 5. The small error along $X$ axis can be observed in this figure, besides of the small error between each step. However, this error is negligible. When the robot performs a gait during long time, e.g., when it is performing some humanitarian demining task (landmine searching), the summation of this error can be compensated normally.

Figure 6 shows a photographic sequence of gait pattern of the hexapod robot. In this Figure the scanning manipulator arm is executing a search strategy. 


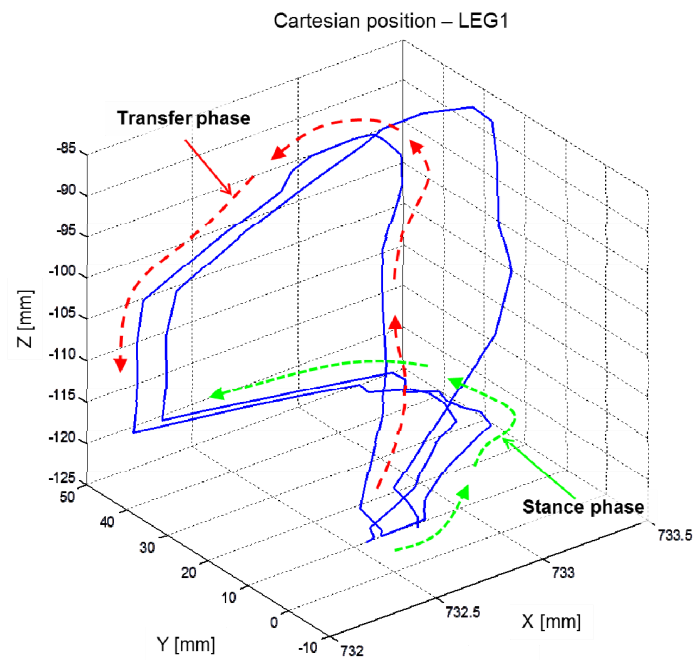

Figure 5. Phases of transfer and stance of the leg 1.

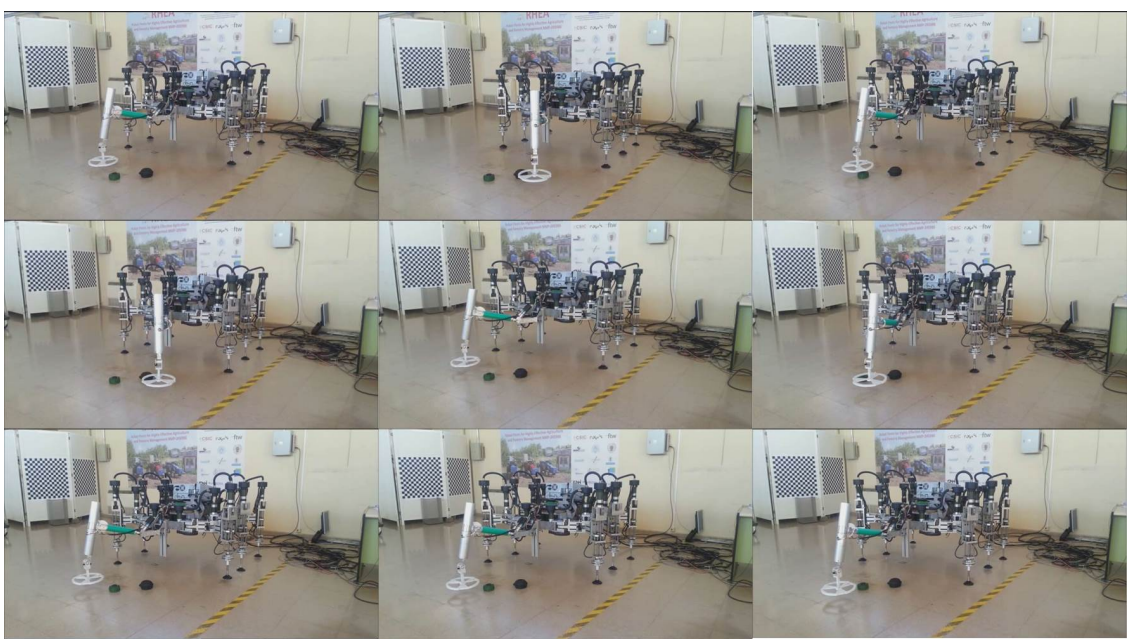

Figure 6. Photographic sequence (left to right and up to down) of a gait pattern of the hexapod robot.

\section{Conclusions}

In this paper an inspection platform (hexapod walking robot) for applications for humanitarian demining tasks has been presented. This platform will support onboard a scanning manipulator arm and other sensors to detect and localize antipersonnel mines, firstly, within a controlled environment. After several tests, it 
will attempt to use this inspection platform in a real environment with surrogate landmines.

\section{Acknowledgements}

The authors acknowledge funding of this work under TIRAMISU Project Grant Agreement $\mathrm{N}^{\circ} 284747$ of the 7FP, and partial funding from the RoboCity2030-III-CM project (Robótica aplicada a la mejora de la calidad de vida de los ciudadanos. Fase III; S2013/MIT-2748), funded by Programas de Actividades I+D en la Comunidad de Madrid and cofunded by Structural Funds of the EU. Dr. Héctor Montes also acknowledges support from Universidad Tecnológica de Panamá.

\section{References}

1. Y. Baudoin et al., CLAWAR, Year 2 Report: TASK 9, WS-HUDEM (1999).

2. Landmine Monitor (2010)

3. Landmine Monitor (2011)

4. Y. Baudoin and I. Doroftei, In Proc. 6th IARP WS-HUDEM, 93 (2012).

5. Y. Baudoin, M. Habib, I. Doroftei, Woodhead Publishing Limited, (2011).

6. P. Kopacek and L. Silberbauer, 7th IARP WS-HUDEM, (2008).

7. E. Fukushima, M. Freese, T. Matsuzawa, T. Aibara, and S. Hirose, Intl. J. Smart Sensing and Intelligent Sys., 1(3), 735 (2008)

8. K. Munsang et al., IARP WS-HUDEM, (2002).

9. D. Waterman, K. Nonami, R. Yuasa, S. Amano, S. Masunaga, H. Ono, IARP WS-HUDEM, (2005).

10. P. Gonzalez and M.A. Jimenez, J. of Robotics Sys., 12(9): 599 (1995)

11. J.C. Habumuremyi et al., CLAWAR'98, (1998)

12. S. Hirose and K. Kato, In Proc. CLAWAR'98, 261 (1998),

13. P. Gonzalez de Santos, E. Garcia, J. Estremera, and M. Armada, M.A. IARP WS-HUDEM, (2002).

14. K. Nonami et al., JSME Intl. Journal, Series C46(3), 881 (2003)

15. P. Gonzalez de Santos, J. Cobano, E. Garcia, J. Estremera, M. Armada, Mechatronics, 17: 417 (2007).

16. L. Marques, M. Rachkov and A.T. Almeida, In Proc. 10th Mediterranean Conf. Control and Autom., (2002)

17. H. Montes, L. Mena, R. Fernández, J. Sarria, P. González de Santos, M. Armada, Proc. RISE 2015, 8th IARP, (2015)

18. R. Fernández, H. Montes, C. Salinas, P. González de Santos, M. Armada, Industrial Robot: An Intl. Journal, 39(5), 450 (2012). 